\title{
Analysis of primary and secondary squamous cell carcinoma of the thyroid gland: a retrospective study
}

\author{
Gang Liu ${ }^{1,2}$, Xiequn $\mathrm{Xu}^{1}$, Ge Chen ${ }^{1}$, Ziwen Liu ${ }^{1}$ \\ ${ }^{1}$ Department of General Surgery, Peking Union Medical College Hospital, Peking Union Medical College and Chinese Academy of Medical \\ Sciences, Beijing, China; ${ }^{2}$ Department of Breast Surgical Oncology, Cancer Hospital of Chinese Academy of Medical Sciences, Beijing, China \\ Contributions: (I) Conception and design: G Liu, X Xu; (II) Administrative support: X Xu, G Chen, Z Liu; (III) Provision of study materials \\ or patients: X Xu, G Chen, Z Liu; (IV) Collection and assembly of data: G Liu, X Xu; (V) Data analysis and interpretation: G Liu, X Xu; (VI) \\ Manuscript writing: All authors; (VII) Final approval of manuscript: All authors. \\ Correspondence to: Xiequn Xu, MD. Department of General Surgery, Peking Union Medical College Hospital, Peking Union Medical College and \\ Chinese Academy of Medical Sciences, No.1 Shuaifuyuan, Dongcheng District, Beijing 100730, China. Email: xxq75@163.com.
}

Background: Squamous cell carcinoma of the thyroid (SCCT) is a kind of rare malignant disease. This study aimed to analyse the clinical characteristics of patients with primary SCCT (PSCCT) and secondary SCCT (SSCCT).

Methods: The medical records of seventeen patients with PSCCT and six patients with SSCCT were reviewed. Clinical symptoms, ultrasound, and pathological characteristics were compared between the two groups.

Results: The mean age of diagnosis was approximately 56 years old in each group. The most common chief complaint of the patients was neck mass in both groups. The percentage of patients with cough $(\mathrm{P}=0.008)$ or weight loss $(\mathrm{P}=0.021)$ was higher in the SSCCT group than the PSCCT group. The mean size of the tumor in the PSCCT group was larger than that in the SSCCT group $(3.382 \pm 2.011$ vs. $1.950 \pm 0.794 \mathrm{~cm}, \mathrm{P}=0.024)$. Papillary thyroid carcinoma (PTC) could be combined with or recur as PSCCT. Kaplan-Meier analysis indicated that the mean survival times after diagnosis were 17.053 months in the PSCCT patients and 13.500 months in the SSCCT patients. Multivariate survival analysis using a Cox regression model demonstrated that predictors of overall survival (OS) in PSCCT patients included age $(\mathrm{P}=0.020)$, enlarged cervical lymph node $(\mathrm{P}=0.017)$, radical operation $\left(\mathrm{P}=4.82 \times 10^{-3}\right)$, and $\mathrm{PTC}(\mathrm{P}=0.03)$.

Conclusions: SCCT is aggressive, with neck mass being the most common chief complaint. There were several different characteristics between PSCCT and SSCCT. PTC could be combined with or recur as PSCCT. Age, enlarged cervical lymph node, radical operation, and PTC were the predictors of OS in PSCCT patients.

Keywords: Thyroid; squamous cell carcinoma; cancer; head and neck; papillary thyroid carcinoma (PTC)

Submitted Jul 25, 2020. Accepted for publication Nov 11, 2020.

doi: 10.21037 /gs-20-628

View this article at: http://dx.doi.org/10.21037/gs-20-628

\section{Introduction}

Squamous cell carcinoma of the thyroid (SCCT) is a rare malignant disease and includes primary squamous cell carcinoma of the thyroid (PSCCT) and secondary squamous cell carcinoma of the thyroid (SSCCT) based on the origination. PSCCT is a rare carcinoma, constituting less than $1 \%$ of all thyroid malignancies (1). It was first reported by von Karst in 1958 (1,2). Although half a century has passed, the etiology of PSCCT is still unknown. Some researchers have indicated that the squamous cells originated from metaplasia complicated with chronic thyroid diseases (3). Some other studies reported that the squamous epithelium originated from the ultimobranchial 
bodies or the thyroglossal duct (4). The PSCCT patients are always diagnosed in the fifth or sixth decades of life (5-7). Most related studies suggest that PSCCT is an aggressive cancer, and most patients die of airway compromise $(1,6,7)$. Age, tumor grade, and tumor size are related to overall survival (OS) and disease-specific survival (DSS) (5). Early diagnosis, radical operation, and adjuvant radiation therapy seem to be associated with a better outcome (6). SSCCT is a type of squamous cell carcinoma that metastasizes to the thyroid. Although there are only a few reports of SSCCT, it is postulated that adjacent organs, including the esophagus, trachea, and larynx, are the most common primary sites $(8,9)$. Some studies have demonstrated that SSCCT is more commonly seen and has a better outcome than PSCCT $(7,8)$. One of the principles for distinguishing SSCCT from PSCCT is to find the primary tumor. Thyroid transcription factor (TTF) and thyroglobulin of the pathology could also help to differentiate those two diseases (7). Because of their scarcity, there have only been a few reports of SCCT in the world, and most of them were reviews of several databases. Until now, there have been few studies with more details of the clinical manifestations. It is very valuable to predict the prognosis based on the clinical manifestations. In this study, we reviewed the clinical characteristics of SCCT patients in China. We compared the clinical symptoms and ultrasound and pathological characteristics, aiming to gain a better understanding of the diagnosis and prognosis of SCCT. We present the following article in accordance with the STROBE reporting checklist (available at http://dx.doi. org/10.21037/gs-20-628).

\section{Methods}

\section{Patients and materials}

Patients diagnosed with PSCCT and patients diagnosed with SSCCT were enrolled at the Peking Union Medical College Hospital (PUMCH) from March 1992 to October 2019. The inclusion and exclusion criteria of PSCCT were as follows $(5,10)$.

Inclusion criteria: (I) patients diagnosed as PSCCT with pathological evidence from biopsy or resection specimen; (II) patients with complete medical records. Exclusion criteria: (I) patients with known primary cancer of other sites; (II) patients with incomplete medical records.

All the SSCCT patients were diagnosed as thyroid metastases from other primary sites. Clinical information was collected from the medical records, including gender, age, carcinoma origination, chief complaint, weight loss, complicated chronic disease, cigarette use, related physical examination, ultrasound, and laryngoscope manifestation. Information including treatment, pathological signs and outcome was also collected. Well-differentiated, moderately differentiated and poorly differentiated classification of Squamous cell carcinoma (SCC) was used to estimate the differentiation grade of SCCT. All of the patients were reviewed by at least two experienced surgeons. The study was conducted in accordance with the Declaration of Helsinki (as revised in 2013). The study was approved by the Ethical Committee of the Peking Union Medical College Hospital (No. JS-2555) and individual consent for this retrospective analysis was waived.

\section{Statistical analysis}

Data were analysed using SPSS software (version 16.0, SPSS Inc., Chicago, IL). According to their characteristics, the differences were evaluated using the Fisher test, the $\chi^{2}$ test, or the independent samples $t$-test. An OS curve was constructed using the Kaplan-Meier method. Univariate survival analysis was calculated via the log-rank test. Factors with log-rank $\mathrm{P}$ values less than 0.15 in the univariate analysis were selected for multivariate survival analysis using a Cox regression model. Patients with missing data were excluded in each subgroup analysis. $\mathrm{P}$ values less than 0.05 were considered statistically significant.

\section{Results}

\section{Basic clinical characteristics}

There were 17 patients diagnosed with PSCCT (8 males and 9 females; median age $56.059 \pm 14.889$ years; ranging from 31 to 83 years) and 6 patients diagnosed with SSCCT (5 males and 1 female; median age $56.833 \pm 9.304$ years; ranging from 45 to 67 years) (Table 1). There were no significant differences in gender and age between the two groups. The primary sites of SSCCT were the esophagus (33\%), larynx (17\%), hypopharynx (17\%) and lung/trachea $(33 \%)$.

\section{Symptoms and imaging characteristics}

The most common chief complaint was neck mass in both groups (53\% in PSCCT group and 67\% in SSCCT group) (Table 1). Approximately $47 \%$ of the PSCCT patients also 
Table 1 Basic characteristics, clinical symptoms, physical examination, and ultrasound characteristics of the 23 patients with SCCT

\begin{tabular}{|c|c|c|}
\hline Characteristic & PSCCT $(n=17)$ & $\operatorname{SSCCT}(n=6)$ \\
\hline $\begin{array}{l}\text { Gender (male:female, } \\
\text { percentage of female) }\end{array}$ & $8: 9(53 \%)$ & $5: 1(17 \%)$ \\
\hline Mean age of diagnosis & $56.059 \pm 14.889$ & $56.833 \pm 9.304$ \\
\hline \multicolumn{3}{|l|}{ Carcinoma origination } \\
\hline Thyroid & $17(100 \%)$ & 0 \\
\hline Esophagus & 0 & $2(33 \%)$ \\
\hline Larynx & 0 & $1(17 \%)$ \\
\hline Hypopharynx & 0 & $1(17 \%)$ \\
\hline Lung/trachea & 0 & $2(33 \%)$ \\
\hline \multicolumn{3}{|l|}{ Chief complaint } \\
\hline Neck mass & $9(53 \%)$ & $4(67 \%)$ \\
\hline Hoarseness & $8(47 \%)$ & $4(67 \%)$ \\
\hline Dyspnea & $5(29 \%)$ & $2(33 \%)$ \\
\hline Cough* & $1(6 \%)$ & $4(67 \%)$ \\
\hline Weight loss* & $2(12 \%)$ & $4(67 \%)$ \\
\hline Chronic disease & $9(53 \%)$ & $5(83 \%)$ \\
\hline Cigarette use & $5(29 \%)$ & $5(83 \%)$ \\
\hline \multicolumn{3}{|l|}{ Physical examination } \\
\hline Enlarged cervical LN & $4(24 \%)$ & $3(50 \%)$ \\
\hline \multicolumn{3}{|l|}{ Ultrasound } \\
\hline Hypoechoic & $11 / 15(73 \%)$ & $4 / 5(80 \%)$ \\
\hline Scattered hyperechoic & $9 / 15(60 \%)$ & $1 / 5(20 \%)$ \\
\hline Blood flow signal & $9 / 15(60 \%)$ & $2 / 5(40 \%)$ \\
\hline Thyroid diffuse lesions & $1 / 15(7 \%)$ & $1 / 5(20 \%)$ \\
\hline \multicolumn{3}{|l|}{ Laryngoscope } \\
\hline UVCP & $4 / 8(50 \%)$ & $1 / 4(25 \%)$ \\
\hline BVCP & $1 / 8(13 \%)$ & 0 \\
\hline Basically normal & 3/8 (38\%) & $3 / 4(75 \%)$ \\
\hline
\end{tabular}

*, $\mathrm{P}$ values of the difference were significant within groups; ", patients complicated with chronic disease including hypertension, diabetes mellitus, coronary artery disease, and so on. PSCCT, primary squamous cell carcinoma of the thyroid; SSCCT, secondary squamous cell carcinoma of the thyroid; $\mathrm{M}$, male; F, female; CLN, cervical lymph node; UVCP, unilateral vocal cord paralysis; BVCP, bilateral vocal cords paralysis. complained of hoarseness, and $67 \%$ of the SSCCT patients complained of hoarseness and cough. The percentage of patients with cough was higher in the SSCCT group than in the PSCCT group $(\mathrm{P}=0.008)$. Approximately $29 \%$ of the PSCCT patients and $33 \%$ of the SSCCT patients complained of dyspnea when they came to the hospital. Weight loss was a complaint of $12 \%$ of patients in the PSCCT group and $67 \%$ of patients in the SSCCT group $(\mathrm{P}=0.021)$. Approximately $53 \%$ of PSCCT patients and $83 \%$ of SSCCT patients were complicated with chronic diseases, such as hypertension, diabetes mellitus, coronary artery disease. A total of $29 \%$ of PSCCT patients and $83 \%$ of SSCCT patients used cigarettes. Approximately $24 \%$ of PSCCT patients and 50\% of SSCCT patients had enlarged cervical lymph nodes during the physical examination in the hospital.

Two of the PSCCT patients and one of the SSCCT patients had no complete ultrasound examination results. According to the ultrasound, most of the lesions were hypoechoic in both groups. Approximately $60 \%$ of lesions in the PSCCT patients were scattered hyperechoic and had blood flow signals, while $20 \%$ of lesions in the SSCCT patients were scattered hyperechoic, and $40 \%$ of lesions had blood flow signals. Thyroid diffuse lesions could also be seen in 7\% PSCCT patients and 20\% SSCCT patients. Eight of the PSCCT patients and four of the SSCCT patients underwent laryngoscope examination. Based on the laryngoscopy results, vocal cord paralysis was more commonly seen in PSCCT patients (Table 1).

\section{Treatment and pathological features}

In the PSCCT patients, $24 \%$ underwent a palliative operation, and $76 \%$ underwent a radical operation (Table 2). In the patients with a radical operation, three relapsed and could only undergo a palliative operation. In the SSCCT patients, $50 \%$ underwent a radical operation, and $50 \%$ underwent a palliative operation.

According to the pathological characteristics, we found that the mean size of the tumor in the PSCCT group was $3.382 \pm 2.011 \mathrm{~cm}$, and the mean size of the tumor in the SSCCT group was $1.950 \pm 0.794 \mathrm{~cm}$. The difference in size was significant $(\mathrm{P}=0.024)$. We also compared the grade of each carcinoma, four PSCCT patient were excluded because of difficulty with classification. We found that 
Table 2 Treatment, pathological characteristics, and outcome of the 23 patients with thyroid squamous cell carcinoma

\begin{tabular}{|c|c|c|}
\hline Characteristics & PSCCT & SSCCT \\
\hline \multicolumn{3}{|l|}{ Treatment } \\
\hline Palliative operation & $4 / 17(24 \%)$ & $3 / 6(50 \%)$ \\
\hline Radical operation\# & 13/17 (76\%) & $3 / 6(50 \%)$ \\
\hline \multicolumn{3}{|l|}{ Pathological characteristics } \\
\hline Mean size of the tumor $(\mathrm{cm})^{*}$ & $3.382 \pm 2.011$ & $1.950 \pm 0.794$ \\
\hline \multicolumn{3}{|l|}{ SCC grade } \\
\hline Well-differentiated & $1 / 13(8 \%)$ & 2/6 (33\%) \\
\hline Moderately differentiated & $4 / 13(31 \%)$ & 1/6 (17\%) \\
\hline Poorly differentiated & $8 / 13(61 \%)$ & $3 / 6(50 \%)$ \\
\hline Lymph node metastasis & $10 / 17(59 \%)$ & $3 / 6(50 \%)$ \\
\hline Metastasis & $7 / 17(41 \%)$ & $6 / 6(100 \%)$ \\
\hline Thyroid & NA & $6 / 6(100 \%)$ \\
\hline Lung & $5 / 17(29 \%)$ & $1 / 6(17 \%)$ \\
\hline Bone & $4 / 17(24 \%)$ & 0 \\
\hline Esophagus & $3 / 17(18 \%)$ & 0 \\
\hline Trachea & $2 / 17(12 \%)$ & 0 \\
\hline Liver & $2 / 17(12 \%)$ & 0 \\
\hline Brain & $1 / 17(5 \%)$ & 0 \\
\hline SCC with PTC & $6 / 17(35 \%)$ & 0 \\
\hline
\end{tabular}

*, $\mathrm{P}$ value of the difference was significant within groups. ", including the palliative operation of relapsed lesion after radical operation. Palliative operation, including tracheotomy, biopsy without radical resection. NA, not available; PSCCT, primary squamous cell carcinoma of the thyroid; SSCCT, secondary squamous cell carcinoma of the thyroid; SCC, squamous cell carcinoma; PTC, papillary thyroid carcinoma.

there was a lower percentage of well-differentiated grade in the pathology of the PSCCT group (8\%) than in the SSCCT group (33\%). More than half of the patients had lymph node metastasis combed with clinical manifestations. Although we excluded other primary sites of the PSCCT patients at diagnosis, we also found that the PSCCT could metastasize to the lung (29\%), bone (24\%), esophagus $(18 \%)$, trachea $(12 \%)$, liver $(12 \%)$ and brain $(5 \%)$ in the follow-up. Furthermore, we found that six PSCCT patients (36\%) had papillary thyroid carcinoma (PTC) (Figure 1). Moreover, two of them had two carcinomas at the same time, and four of them found SCCT after recurrent PTC, which may support the hypothesis that the squamous cells originated from a metaplasia complicated with chronic thyroid diseases.

According to Kaplan-Meier survival analysis, the mean survival times after diagnosis were $17.053 \pm 3.361$ months

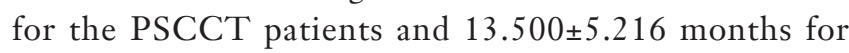
the SSCCT patients (Figure 2). There was no significant difference between these two groups $(\mathrm{P}=0.843)$. The mean survival times were 17.053 months (95\% CI: 10.466-23.641) in the PSCCT patients and 13.500 months (95\% CI: 3.276-23.724) in the SSCCT patients. The median survival times were 12 months (95\% CI: 8.137-15.863) in the PSCCT patients and 3 months (95\% CI: 0.00-12.602) in the SSCCT patients. We also conducted univariate survival analysis of variables using the Kaplan-Meier method in each group. The survival time was significantly different based on age (log-rank $\left.\mathrm{P}=1.80 \times 10^{-4}\right)$, radical operation (log-rank $\mathrm{P}=0.005)$, and tumor size $\left(\log\right.$-rank $\left.\mathrm{P}=1.68 \times 10^{-4}\right)$ in PSCCT (Table 3). The survival time manifested with a statistically significant difference based on cough (log-rank $\mathrm{P}=0.045$ ), and weight loss (log-rank $\mathrm{P}=0.045)$ in SSCCT.

In the multivariate analysis, we assessed the factors with log-rank p values less than 0.15 in the univariate analysis. Using a Cox regression model, we found that the following factors were independent predictors of PSCCT OS: age at diagnosis (HR 1.251, 95\% CI: 1.036-1.510, $\mathrm{P}=0.020$ ), enlarged cLN of PE (HR 47.560, 95\% CI: 1.995-1133.772, $\mathrm{P}=0.017$ ), radical operation (HR $1.06 \times 10^{-3}, 95 \% \mathrm{CI}: 9 \times 10^{-6}-$ $0.124, \mathrm{P}=4.82 \times 10^{-3}$ ), and PTC (HR 34.448, 95\% CI: 1.402 846.333, $\mathrm{P}=0.030$ ) (Table 4). There were no significant independent predictors of OS in the SSCCT patients by multivariate survival analysis.

\section{Discussion}

SCCT is an aggressive malignant disease that includes PSCCT and SSCCT. Bothe types have an extremely poor prognosis $(11,12)$. PSCCT is rarely seen in the clinic, and only hundreds of cases have been reported in the literature, most of which were from the review of databases. Along with the increasing frequency of thyroid regular examination, SSCCT could be a type of metastatic malignancy mimicking PSCCT.

In this study, we reviewed the clinical data of PSCCT and SSCCT patients in China. Most of the patients were approximately 50 to 60 years old in both groups. According to previous studies, most PSCCT patients were more than 60 years old $(5,6,11)$. The percentage of female PSCCT patients was slightly higher than that of males PSCCT 

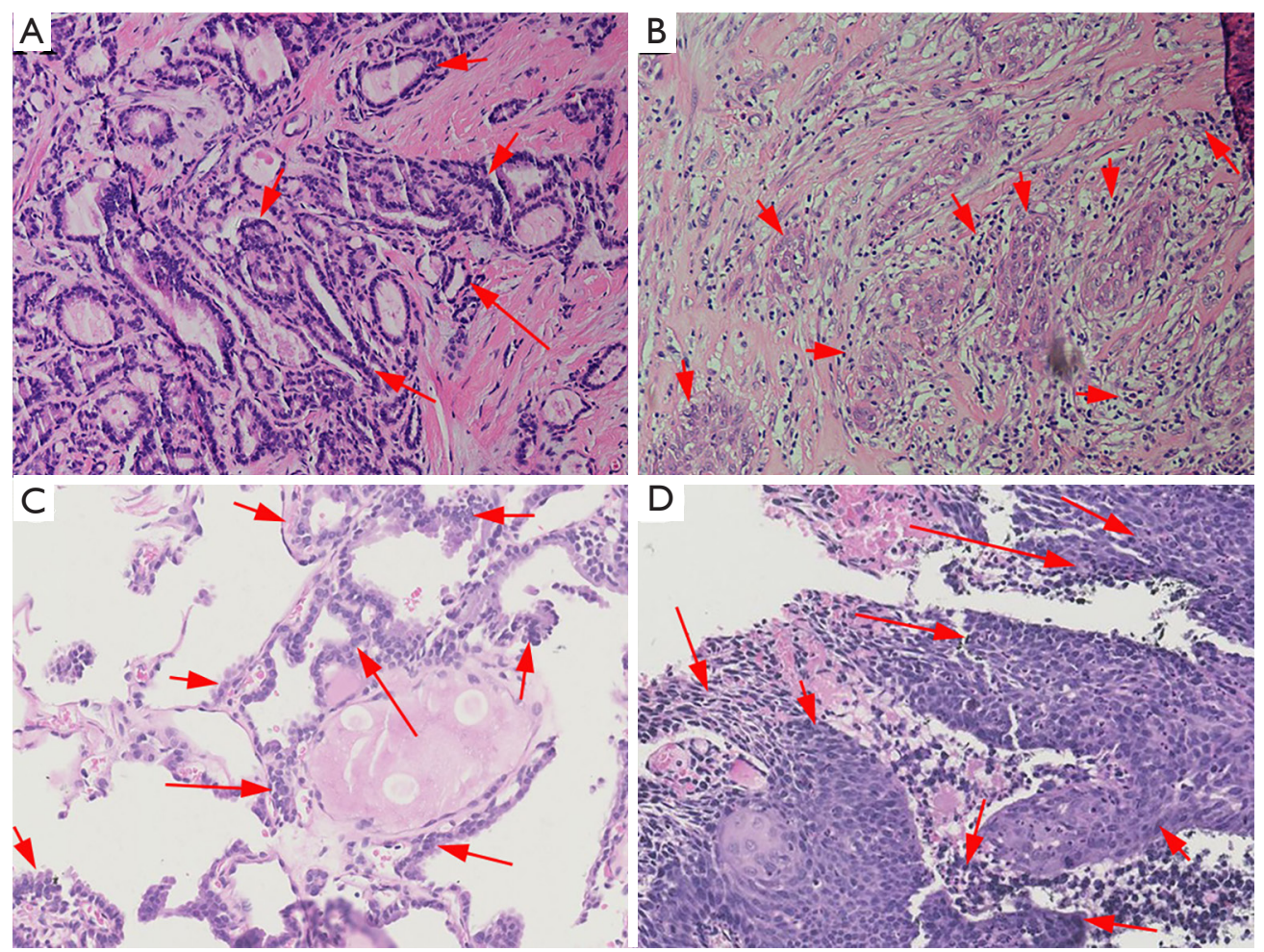

Figure 1 Histopathology of the PSCCT patients with PTC $(\mathrm{HE}, \times 200)$. (A) PTC part in the resection specimen; (B) squamous cell carcinoma part in the resection specimen; (C) PTC part in the fine-needle aspiration biopsy; (D) squamous cell carcinoma part in the fine-needle aspiration biopsy. PSCCT, primary squamous cell carcinoma of the thyroid; PTC, papillary thyroid carcinoma. Arrows marked recommended observation position.

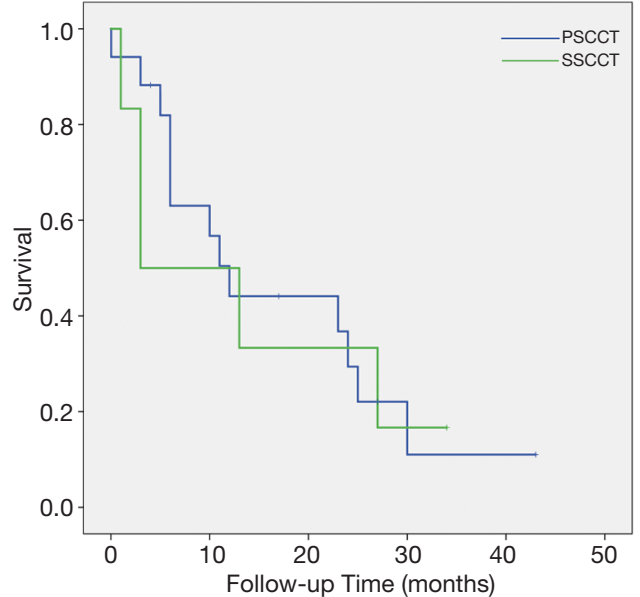

Figure 2 Kaplan-Meier survival curve of SCCT. SCCT, squamous cell carcinoma of the thyroid. patients in our study, which is consistent with previous studies $(5,6,11)$. Since the sample size of our study was limited, further related studies with larger sizes are needed to identify the tendency of gender, especially in SSCCT. We also identified the primary sites of involvement in SSCCT patients. Most of the primary sites were from the adjacent organs from the neck. From the literature review, carcinoma from other organs could also metastasize to the thyroid, including the colon, skin, kidney, uterus, stomach, bone marrow, and prostate (8). However, the squamous cell carcinoma cell type could only be seen in the lung and other organs from the head and neck $(8,9)$.

We also found that neck mass was the most common chief complaint among PSCCT and SSCCT patients in our study, which might be caused by the rapid enlargement of the carcinoma. Hoarseness were also commonly seen in 
Table 3 Univariate survival analysis of variables using Kaplan-Meier method in each group

\begin{tabular}{lcc}
\hline \multirow{2}{*}{ Characteristic } & \multicolumn{2}{c}{ Log-rank P value } \\
\cline { 2 - 3 } & PSCCT & SSCCT \\
\hline Gender & 0.972 & 0.665 \\
Age & $1.80 \times 10^{-4}$ & 0.118 \\
Neck mass & 0.068 & 0.062 \\
Hoarseness & 0.655 & 0.770 \\
Dyspnea & 0.119 & 0.062 \\
Cough & 0.335 & 0.045 \\
Weight loss & 0.416 & 0.045 \\
Chronic disease & 0.971 & 0.481 \\
Cigarette use & 0.559 & 0.897 \\
Enlarged cLN of PE & 0.091 & 0.289 \\
Hypoechoic of ultrasound & 0.530 & 0.221 \\
Scatter hyperechoic of ultrasound & 0.758 & 0.695 \\
Blood flow signal of ultrasound & 0.397 & 0.289 \\
Diffused lesion of ultrasound & 0.470 & 0.221 \\
UVCP & 0.935 & 0.317 \\
VC Basically normal & 0.628 & 0.317 \\
Radical operation & 0.005 & 0.134 \\
Blood transfusion & 0.718 & 0.405 \\
Tumor size & $1.68 \times 10^{-4}$ & 0.754 \\
SCC grade & 0.264 & 0.062 \\
LN metastasis & 0.163 & 0.289 \\
Metastasis & 0.397 & $\mathrm{NA}$ \\
\hline SCC with PTC & 0.124 & $\mathrm{NA}$ \\
\hline
\end{tabular}

PSCCT, primary squamous cell carcinoma of the thyroid; SSCCT, secondary squamous cell carcinoma of the thyroid; $\mathrm{PE}$, physical examination; $\mathrm{CLN}$, cervical lymph node; UVCP, unilateral vocal cord paralysis; VC, vocal cords; SCC, squamous cell carcinoma; LN, lymph node; PTC, papillary thyroid carcinoma; NA, not available.

both groups. We hypothesized that the recurrent laryngeal nerve was easily influenced by the malignancy because of its immediate vicinity and the tumor's invasiveness. Cough and weight loss were more commonly seen in SSCCT patients, which we hypothesized were determined by the primary site of the carcinoma. The symptoms and physical examinations of SCCT patients revealed distinguishing features, and ultrasound could provide some help in diagnosis. A hypoechoic sign on ultrasound was the most common feature of the lesions. Some of the PSCCT lesions also manifested with scattered hyperechoic signal and blood flow signal. Previous studies also indicated that eggshell calcification could be seen in the sonographic findings of PSCCT (13).

Our study results also showed that the pathological characteristics and outcomes were poorer in the PSCCT patients. Several PSCCT patients relapsed and could only undergo a palliative operation, even after a radical operation. The tumor size was larger, the SCC grade was lower, and more patients with PSCCT had lymph node metastases. In accordance with literature studies, we confirmed that the PSCCT could metastasize to other organs due to the invasiveness of PSCCT $(6,14)$.

Consistent with previous reports, our study also confirmed that PTC could recur as SCCT $(12,15)$. Approximately $35 \%$ of PSCCT patients had PTC, and twothirds of them were diagnosed with PSCCT after recurrent PTC. Thus, regular examination is necessary for patients with PTC, especially for those with recurrence.

We found that the prognosis of SCCT patients was poor, with a short median survival time, although SSCCT is a type of metastatic disease, and its malignancy mainly depends on the primary site. Previous studies also found that PSCCT is more aggressive than SSCCT (7). Our study indicated that the mean and median survival times were longer in PSCCT patients than in SSCCT patients. We also found that age at diagnosis, enlarged cervical lymph nodes in the physical examination, and PTC were independent risk predictors of OS in PSCCT. Radical operation was a protective independent predictor of OS in PSCCT. Further research is needed to explore the underlying mechanism. As this study was limited to the sample size of SSCCT patients, no independent predictors were found in SSCCT using multivariate survival analysis.

There are several limitations in our study. First, according to the literature, additional tests could improve the diagnosis rate, such as BRAF mutation testing (16), PET/CT (17), and immunoreactions including p21, MIB-I, p53, thyroglobulin and TTF $(7,18)$. Although we excluded other primary sites of PSCCT using CT, laryngoscope, and upper gastrointestinal contrast at diagnosis, more techniques should be added to improve the accuracy. Second, the number of patient sample was small. The significance of this analysis could be influenced as a result, especially for the SSCCT group. Thus, larger samples are 
Table 4 Multivariate survival analysis using Cox regression model in the PSCCT group

\begin{tabular}{|c|c|c|c|c|c|}
\hline Characteristic & $\mathrm{B}$ & SE & $P$ value & HR & $95 \% \mathrm{Cl}$ \\
\hline Neck mass & 1.190 & 1.552 & 0.443 & 3.287 & $0.157-68.817$ \\
\hline Dyspnea & 0.391 & 0.918 & 0.671 & 1.478 & $0.244-8.943$ \\
\hline Enlarged cLN of PE & 3.862 & 1.618 & 0.017 & 47.560 & $1.995-1,133.772$ \\
\hline Tumor size & -0.507 & 0.352 & 0.150 & 0.602 & $0.302-1.201$ \\
\hline PTC & 3.539 & 1.633 & 0.030 & 34.448 & $1.402-846.333$ \\
\hline
\end{tabular}

PE, physical examination; PTC, papillary thyroid carcinoma; $c L N$, cervical lymph node; $\mathrm{HR}$, hazard ratio; Cl, confidential interval.

needed for replication.

\section{Conclusions}

In conclusion, we described the largest cohort of PSCCT in one single center in China. SCCT is aggressive, with neck mass being the most common chief complaint. There were several different characteristics between PSCCT and SSCCT. A hypoechoic signal with a scattered hyperechoic sign of ultrasound supports the diagnosis. PTC could be combined with or recurrent as PSCCT. Age, enlarged cervical lymph nodes, radical operation, and PTC were the predictors of OS in PSCCT patients. These characteristics can help elucidate the diagnosis and prognosis of SCCT.

\section{Acknowledgments}

We give our great thanks to the patients for their active participation.

Funding: This research was funded in part by the National Natural Science Foundation of China (81902271 to G Liu).

\section{Footnote}

Reporting Checklist: The authors have completed the STROBE reporting checklist. Available at http://dx.doi. org/10.21037/gs-20-628

Data Sharing Statement: Available at http://dx.doi. org/10.21037/gs-20-628

Conflicts of Interest: All authors have completed the ICMJE uniform disclosure form (available at http://dx.doi. org/10.21037/gs-20-628). The authors have no conflicts of interest to declare.

Ethical Statement: The authors are accountable for all aspects of the work in ensuring that questions related to the accuracy or integrity of any part of the work are appropriately investigated and resolved. The study was conducted in accordance with the Declaration of Helsinki (as revised in 2013). The study was approved by the Ethical Committee of the Peking Union Medical College Hospital (No. JS-2555) and individual consent for this retrospective analysis was waived.

Open Access Statement: This is an Open Access article distributed in accordance with the Creative Commons Attribution-NonCommercial-NoDerivs 4.0 International License (CC BY-NC-ND 4.0), which permits the noncommercial replication and distribution of the article with the strict proviso that no changes or edits are made and the original work is properly cited (including links to both the formal publication through the relevant DOI and the license). See: https://creativecommons.org/licenses/by-nc-nd/4.0/.

\section{References}

1. Huang TY, Assor D. Primary squamous cell carcinoma of the thyroid gland: a report of four cases. Am J Clin Pathol 1971;55:93-8.

2. Zhou XH. Primary squamous cell carcinoma of the thyroid. Eur J Surg Oncol 2002;28:42-5.

3. Goldman RL. Primary squamous cell carcinoma of the thyroid gland: report of a case and review of the literature. Am Surg 1964;30:247-52.

4. Kampsen EB, Jager N, Max MH. Squamous cell carcinoma of the thyroid: a report of two cases. J Surg 
Oncol 1977;9:567-78.

5. Au JK, Alonso J, Kuan EC, et al. Primary Squamous Cell Carcinoma of the Thyroid: A Population-Based Analysis. Otolaryngol Head Neck Surg 2017;157:25-9.

6. Yang S, Li C, Shi X, et al. Primary Squamous Cell Carcinoma in the Thyroid Gland: A Population-Based Analysis Using the SEER Database. World J Surg 2019;43:1249-55.

7. Booya F, Sebo TJ, Kasperbauer JL, et al. Primary squamous cell carcinoma of the thyroid: report of ten cases. Thyroid 2006;16:89-93.

8. Michelow PM, Leiman G. Metastases to the thyroid gland: diagnosis by aspiration cytology. Diagn Cytopathol 1995;13:209-13.

9. Vatsyayan A, Mandlik D, Patel P, et al. Metastasis of squamous cell carcinoma of the head and neck to the thyroid: a single institution's experience with a review of relevant publications. Br J Oral Maxillofac Surg 2019;57:609-15.

10. Lam AK. Squamous cell carcinoma of thyroid: a unique type of cancer in World Health Organization Classification. Endocr Relat Cancer 2020;27:R177-92.

11. Limberg J, Ullmann TM, Stefanova D, et al. Prognostic Characteristics of Primary Squamous Cell Carcinoma of the Thyroid: A National Cancer Database Analysis. World J Surg 2020;44:348-55.

Cite this article as: Liu G, Xu X, Chen G, Liu Z. Analysis of primary and secondary squamous cell carcinoma of the thyroid gland: a retrospective study. Gland Surg 2021;10(2):559-566. doi: $10.21037 / g s-20-628$
12. Dennis K, O'Neil M, Harrington A. Not all neck mass fine-needle aspirations with squamous cells are squamous cell carcinoma; report of a case of recurrent thyroid carcinoma with papillary and squamous components. Cytojournal 2018;15:23.

13. Chen CY, Tseng HS, Lee CH, et al. Primary squamous cell carcinoma of the thyroid gland with eggshell calcification: sonographic and computed tomographic findings. J Ultrasound Med 2010;29:1667-70.

14. Harada T, Shimaoka K, Yakumaru K, et al. Squamous cell carcinoma of the thyroid gland -- transition from adenocarcinoma. J Surg Oncol 1982;19:36-43.

15. Kitahara S, Ito T, Hamatani S, et al. Thyroid papillary carcinoma recurring as squamous cell carcinoma: report of a case. Surg Today 2006;36:171-4.

16. Basnet A, Pandita A, Fullmer J, et al. Squamous Cell Carcinoma of the Thyroid as a Result of Anaplastic Transformation from BRAF-Positive Papillary Thyroid Cancer. Case Rep Oncol Med 2017;2017:4276435.

17. Cai L, Chen Y, Huang Z, et al. Primary squamous cell carcinoma of the thyroid on FDG PET/CT. Clin Nucl Med 2014;39:1014-6.

18. Fassan M, Pennelli G, Pelizzo MR, et al. Primary squamous cell carcinoma of the thyroid: immunohistochemical profile and literature review. Tumori 2007;93:518-21. 